Abstract ID: 26

\title{
Depression symptoms in patients with thyroid disorder at the tertiary hospital in northern region of Malaysia
}

\author{
Siti Nur Illiani Jaafar ${ }^{\mathrm{a}} \mid$ Azlina Daud $^{\mathrm{a}} \mid$ Sanisah Saidi ${ }^{\mathrm{a}}$ | Ramli Musa ${ }^{\mathrm{b}} \mid$ Nik Nur Fatnoon Nik Ahmad \\ ${ }^{a}$ Kulliyyah of Nursing, International Islamic University Malaysia \\ ${ }^{b}$ Kulliyyah of Medicine, International Islamic University Malaysia
}

Introduction: Thyroid disorder patients have a high risk to experience depression symptoms resulting from the thyroid hormone fluctuations. The aims of this study were to evaluate the occurrence of depression symptoms and to analyze the association between depression symptoms and social-demographic of the patients affected by different thyroid disorders. Methods: This was a cross-sectional study of one of the non-profit hospitals in the northern region of Malaysia. Depression symptoms were measured using the Depression Anxiety Stress Scale-21 (DASS-21). Socio-demographic characteristics of the patients were gathered using the questionnaire. Patients with any thyroid disorders, above 18 years old and did not have any psychiatric disorders were included in this study. Results: The total numbers of subjects in this study were 199 . The female consisted of $76.9 \%$ (153). The mean age was $46.32 \pm 15.16$ years. Regarding the thyroid disorder; 35.2\% (70) had hyperthyroidism, $23.6 \%$ (47) had goitre, $14.1 \%$ (28) had hypothyroidism, $14.6 \%$ (29) had autoimmune thyroid disorders while thyroid cancer was found in $12.6 \%$ (25). Determination of depression symptoms showed that $85.9 \%$ (171) had no depression, $6.0 \%$ (12) and 4.5\% (9) had mild to moderate depression respectively while severe depression was found in 3.5\% (7) cases. Age and patients, those not living alone significantly associated with depression symptoms $(p<0.05)$. Conclusions: These findings would suggest that some of the thyroid patients had depression symptoms. In addition, the patients in middle aged and staying with family seem to have depression symptoms. Further studies should be performed, in order to confirm these findings.

KEYWORDS: thyroid, depression symptoms, association factors 